\title{
Binding studies of paeonolum with bovine serum albumin using spectroscopic methods
}

\author{
Chang-yun Chen ${ }^{\mathrm{a}}$, Xiao-tian $\mathrm{Gu}^{\mathrm{b}}$ and Jia-hong Zhou ${ }^{\mathrm{b}, *}$ \\ ${ }^{a}$ Department of Chemistry, Nanjing Xiaozhuang University, Nanjing 210017, PR China \\ ${ }^{\mathrm{b}}$ Analysis \& Detecting Center, Nanjing Normal University, Nanjing 210097, PR China
}

\begin{abstract}
Fluorescence and UV spectroscopic techniques were used to investigate the interaction of Paeonolum, one of the major bioactive components isolated from the bark of peony plant, with bovine serum albumin (BSA). Results obtained reveal that a binding interaction occurs between paeonolum and BSA under physiological conditions, and the interaction can quench the fluorescence of BSA originating from the complex formation between paeonolum and BSA. In addition, according to the thermodynamics parameters of this interaction process, it appears that this binding interaction is mainly hydrophobic in nature.
\end{abstract}

Keywords: Paeonolum, bovine serum albumin, fluorescence quenching, UV absorption spectroscopy

\section{Introduction}

Serum albumin, the most abundant protein in the circulatory system, has been one of the most extensively studied of all proteins [1,2]. It's synthesized in the liver, exported as a non-glycosylated protein, and is present in the blood at about to $40 \mathrm{mg} \cdot \mathrm{ml}^{-1}$ [3]. It's not only the major transport protein unesterified fatty acid, but also capable of binding an extraordinarily diverse range of metabolites, drugs, and organic compounds. Many drugs, including anti-coagulants, tranquilizers, and general anaesthetics, are transported in the blood while bound to albumin [4]. This has stimulated much research on the nature of the drug binding sites and investigation of whether fatty acids, natural metabolites, and drags compete with one another for binding to protein. These studies may provide information of structural features, which determine the therapeutic effectiveness of drugs, and become an important research filed in life sciences, chemistry, and clinical medicine. The molecular interactions are often monitored by using optical techniques which are sensitive and relatively easy to use. For BSA, although different compounds have been probes successfully, such as dyes [5], drugs [6,7], and metal complexes [8,9], the binding of Chinese herbal active component to protein has seldom been investigated [10].

Paeonolum (abbreviated as PAL, whose structure was shown in Fig. 1), one of the major bioactive components isolated from the bark of peony (Chinese name: mudan, which is the national flower of China) is known to have a range of pharmaceutical properties including sedation, hypnosis, antisepsis, anti-inflammatory, anti-oxidant and so on. However, there are no reports in the literature regarding the interaction of PAL with proteins. In this paper, we report the first study to monitor the interaction between PAL and BAS using optical techniques under physiological conditions.

\footnotetext{
${ }^{*}$ Corresponding author: Jiahong Zhou, Associate Professor, Analysis \& Detecting Center, Nanjing Normal University, 122 Ninghai Road, Nanjing, Jiangsu 210097, P.R. China. Fax: +86 258359 8359; E-mail: zhoujiahong@ njnu.edu.cn.
} 


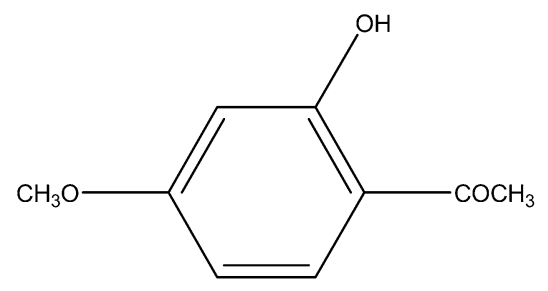

Fig. 1. The structure of PAL.

\section{Materials and methods}

\subsection{Materials and instruments}

Bovine serum albumin (BSA) was purchased from Sigma Company, it was dissolved in the buffer solution containing $0.9 \% \mathrm{NaCl}$, and kept in the dark at $4^{\circ} \mathrm{C}$. Paeonolun was purchased from National Institute for Identification of Pharmaceutical and Biological Products. $\mathrm{NaCl}$ (analytical grade, 0.9\%) solution was used to maintain the ion strength. The buffer solution consists of Tris $\left(0.2 \mathrm{moll}^{-1}\right)$ and $\mathrm{HCl}$ $\left(0.1 \mathrm{moll}^{-1}\right)$, and the $\mathrm{pH}$ was adjusted to 7.40 by adding $0.5 \mathrm{moll}^{-1} \mathrm{NaOH}$.

The absorption spectra were recorded on a TU-1901 spectra-photometer (Beijing) and the fluorescence spectra were recorded on a Perkin Elmer LS-50B spectrofluorophotometer (USA).

\subsection{Experimental methods}

BSA solution was transferred into a $10 \mathrm{ml}$ volumetric flask, then the PAL operating solution was added in different amounts to the flask. The mixture was diluted to a certain volume with doubly distilled water, and mixed thoroughly. After $15 \mathrm{~min}$ the samples were detected by the absorption spectra-photometer and the spectrofluorophotometer.

\section{Results and discussion}

\subsection{UV absorption spectra}

In the region of $200 \mathrm{~nm}$ to $300 \mathrm{~nm}$, BSA has two characteristic absorption bands with absorption maxima at $206 \mathrm{~nm}$ and $278 \mathrm{~nm}$ respectively (Fig. 2), and the main absorption peak is at $206 \mathrm{~nm}$. After adding PAL, notable changes in the absorption spectrum of BSA were observed. Two absorption peaks shifted to longer wavelength slimly, and absorption intensities decreased greatly. The results indicated that there is one binding interaction occur between BSA and PAL.

\subsection{Fluorescence spectra}

Figure 3 shows the emission spectra of BSA in the absence and presence of PAL. As can be seen from Fig. 3, BSA has strong fluorescence emission with a peak at $347 \mathrm{~nm}$ on excitation at $278 \mathrm{~nm}$, and its fluorescence intensity decreases in the presence of PAL. The results indicated that there were strong interactions between the PAL and BSA, it is consistent with the conclusion of absorption spectra. 


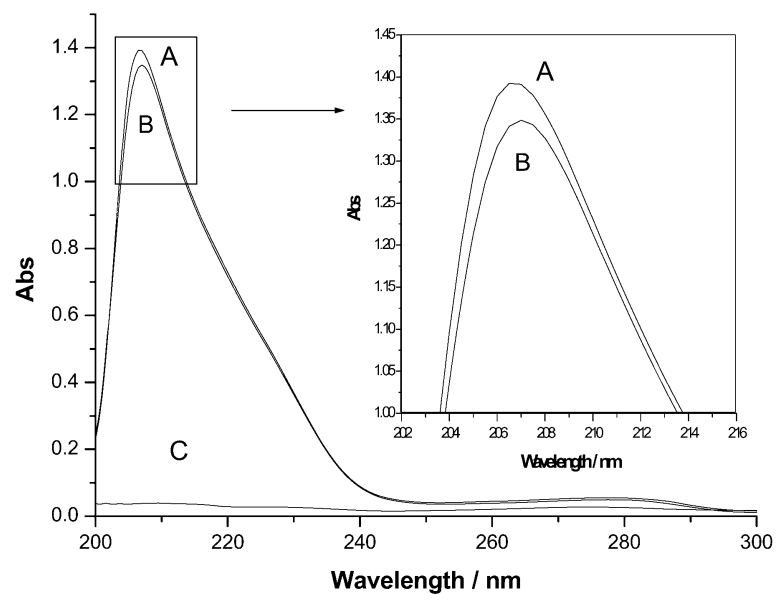

Fig. 2. UV absorption spectra. (A) $(\mathrm{BSA})=9.80 \times 10^{-7} \mathrm{moll}^{-1}$; (B) $9.80 \times 10^{-7} \mathrm{moll}^{-1} \mathrm{BSA}$ in the presence of $9.80 \times 10^{-7} \mathrm{moll}^{-1} \mathrm{PAL}$; (C) $9.80 \times 10^{-7} \mathrm{moll}^{-1} \mathrm{PAL}$.

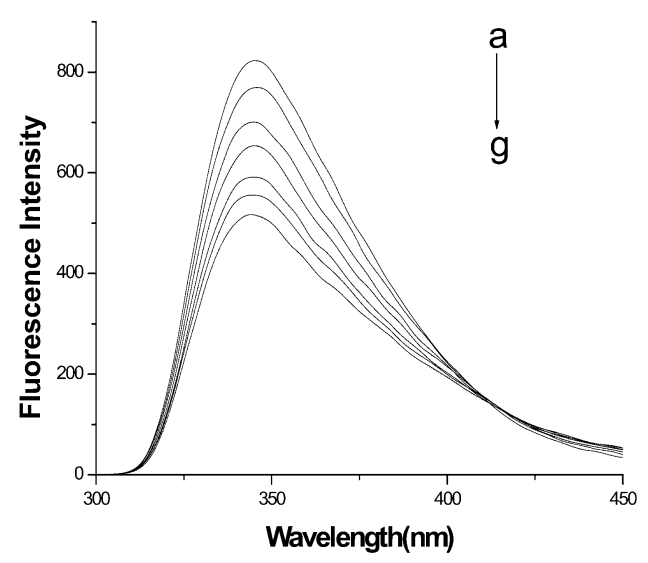

Fig. 3. Fluorescence emission spectra. (a) $9.80 \times 10^{-7} \mathrm{moll}{ }^{-1} \mathrm{BSA}$; (b) to (g), $9.80 \times 10^{-7} \mathrm{~mol} \mathrm{l}^{-1} \mathrm{BSA}$ in the presence of $9.80 \times 10^{-7} \mathrm{moll}^{-1}, 1.96 \times 10^{-6} \mathrm{moll}^{-1}, 2.94 \times 10^{-6} \mathrm{moll}^{-1}, 3.92 \times 10^{-6} \mathrm{moll}^{-1}, 4.90 \times 10^{-6} \mathrm{moll}^{-1}, 5.88 \times 10^{-6} \mathrm{moll}^{-1}$ PAL respectively.

Fluorescence quenching is usually divided into dynamic quenching and static quenching. Dynamic quenching is the interaction between quencher and fluorophore at excited state. Their interactions obey the Stern-Volmer equation:

$$
F_{0} / F=1+K_{q} \tau_{0}[Q]=1+K_{\mathrm{SV}}[Q],
$$

where $F_{0}$ and $F$ are the fluorescence intensities in the absence and presence of PAL, respectively. [Q] is the concentration of PAL and $K_{\mathrm{SV}}$ is the Stern-Volmer quenching constant. $K_{q}$ is the quenching rate constant of the biomolecule, and $\tau_{0}$ is the average lifetime of the biomolecule without quencher. When quencher can form the non-fluorescence complex with fluorophore which is at the ground state, the fluorescence quenching mechanism of this process is static quenching. Generally, the temperature is often used to differentiate between dynamic quenching and static quenching. The quenching constants 
Table 1

The Stern-Volmer constants, quenching rate constants and correlation coefficients $(R)$ of BSA-PAL at different temperatures

\begin{tabular}{cccc}
\hline Temperature $(\mathrm{K})$ & $K_{\mathrm{SV}}\left(\mathrm{l} \mathrm{mol}^{-1}\right)$ & $K_{q}\left(\mathrm{l} \mathrm{mol}^{-1}\right)$ & $R$ \\
\hline 290 & $1.319 \times 10^{5}$ & $1.319 \times 10^{13}$ & 0.9967 \\
300 & $1.192 \times 10^{5}$ & $1.192 \times 10^{13}$ & 0.9990 \\
310 & $1.098 \times 10^{5}$ & $1.098 \times 10^{13}$ & 0.9974 \\
\hline
\end{tabular}

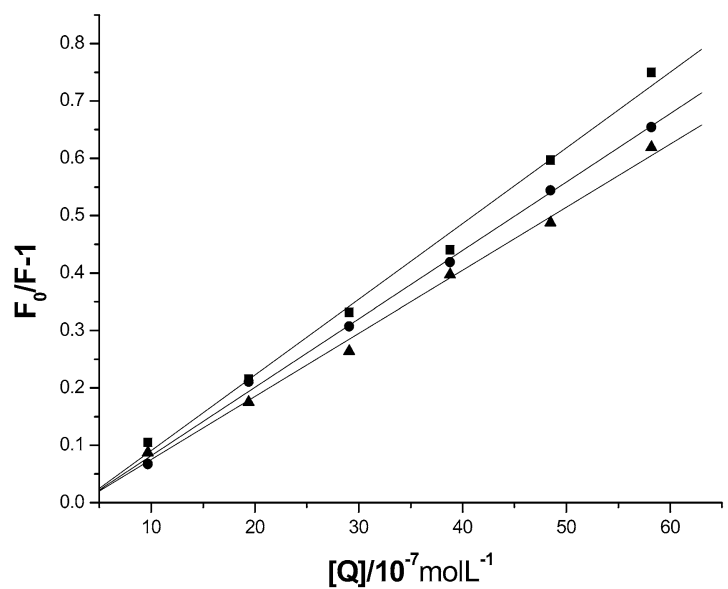

Fig. 4. The Stern-Volmer curves of BSA-PAL. $\left(\lambda_{\mathrm{ex}}=278 \mathrm{~nm}, \lambda_{\mathrm{em}}=347 \mathrm{~nm}, \mathrm{pH}=7.40, C_{\mathrm{BSA}}=9.80 \times 10^{-7} \mathrm{~mol} / 1\right.$, $C_{\mathrm{PAL}}=9.80 \times 10^{-7} \sim 5.88 \times 10^{-6} \mathrm{~mol} / \mathrm{l}$ ) at $290 \mathrm{~K}$ (squares), $300 \mathrm{~K}$ (circles), $310 \mathrm{~K}$ (triangles).

decreased with increased temperature for the static quenching, and the reversed effect was observed for the dynamic quenching. In order to illustrate the fluorescence quenching mechanism of BSA by PAL, the fluorescence intensity data was analyzed according to the Stern-Volmer equation by plotting $F_{0} / F$ versus the PAL concentration $[Q]$ at $290 \mathrm{~K}, 300 \mathrm{~K}$, and $310 \mathrm{~K}$ respectively (see Fig. 4 and Table 1). It can be obviously observed that $K_{\mathrm{SV}}$ decreases while the temperature increasing, therefore, the fluorescence quenching mechanism is static quenching.

Furthermore, the life time of the fluorescence is $10^{-8} \mathrm{~s}$ [11], and the biggest collision quenching rate constant is $2.0 \times 10^{10} 1 \mathrm{~mol}^{-1} \mathrm{~S}^{-1}$ [12]. With the help of $K_{\mathrm{SV}}$ obtained from experiment and Eq. (1), $K_{q}$ can be calculated, and its value is much bigger than $2.0 \times 10^{10} 1 \mathrm{~mol}^{-1} \mathrm{~S}^{-1}$. As a result, it can be further illustrate that the fluorescence quenching mechanism is static quenching also.

\subsection{Binding sites number}

From the equation: $\lg \left[\left(F_{0}-F\right) / F\right]=\lg K_{b}+n \lg [Q]$ [13], the plots of $\lg F_{0} /\left(F_{0}-F\right)$ vs $\lg 1 /[Q]$ are shown in Fig. 5, and binding sites number $n$ can be obtained and listed in Table 2 together with correlation coefficients. From Table 2, it can be observed that temperature has almost no influence on $n$, and the average binding sites number $n$ is one.

It is shown that the binding between PAL and BSA is remarkable and the effect of temperature is small. Thus, PAL can be stored and removed by the proteins in the body. 
Table 2

The binding sites number $\mathrm{n}$ of PAL-BSA at different temperatures

\begin{tabular}{ccc}
\hline Temperature $(\mathrm{K})$ & $n$ & $R$ \\
\hline 290 & 1.09 & 0.9990 \\
300 & 1.09 & 0.9992 \\
310 & 1.09 & 0.9985 \\
\hline
\end{tabular}

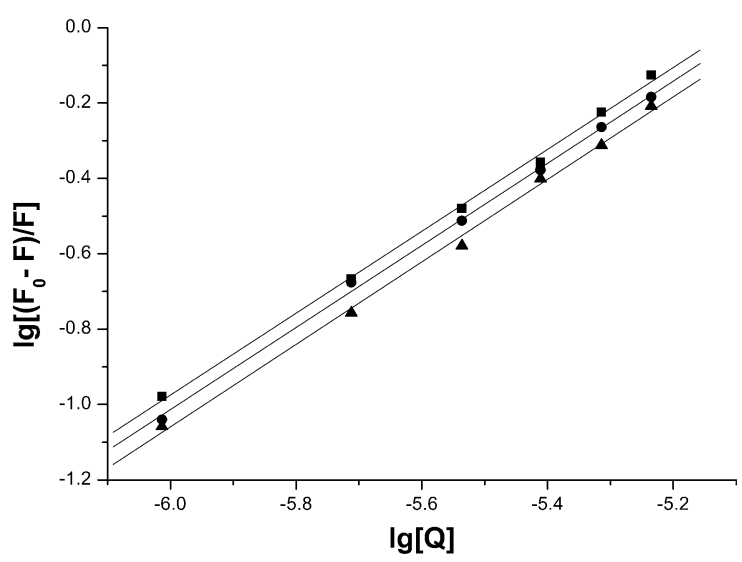

Fig. 5. The binding sites number n of BSA-PAL at $290 \mathrm{~K}$; $300 \mathrm{~K}, 310 \mathrm{~K}$; respectively.

Table 3

Thermodynamic parameters for the binding of PAL to BSA

\begin{tabular}{cccccc}
\hline Temperature $(\mathrm{K})$ & $K \times 10^{5}\left(1 \mathrm{~mol}^{-1}\right)$ & $\Delta G\left(\mathrm{~kJ} \mathrm{~mol}^{-1}\right)$ & $R$ & $\Delta S\left(\mathrm{~J} \mathrm{~mol}^{-1} \mathrm{~K}^{-1}\right)$ & $\Delta H\left(\mathrm{~kJ} \mathrm{~mol}^{-1}\right)$ \\
\hline 290 & 1.319 & -28.44 & & \\
300 & 1.192 & -29.17 & 0.9992 & 74.34 & -6.865 \\
310 & 1.098 & -29.93 & & & \\
\hline
\end{tabular}

\subsection{Binding mode}

The action force between a drug and a bimolecular may include hydrogen bonding, Van der Waals force, electrostatic force and hydrophobic interactions. Because the temperature effect is very small, the interaction enthalpy change can be regarded as a constant if the temperature range is not too wide. Therefore, from the following equations,

$$
\begin{aligned}
& \ln \left(K_{2} / K_{1}\right)=\Delta H\left(1 / T_{1}-1 / T_{2}\right) / R, \\
& \Delta G=\Delta H-T \Delta S=-R T \ln K,
\end{aligned}
$$

where $\Delta H, \Delta G$ and $\Delta S$ are enthalpy change, free energy change and entropy change respectively. These thermodynamics parameters are calculated from the linear relationship between $\ln K$ and the $1 / T$ (Table 3, Fig. 6). $\Delta H$ and $\Delta S$ of the binding interaction between PAL and BSA are calculated to be $-6.8655 \mathrm{~kJ} \mathrm{~mol}^{-1}$ and $74.34 \mathrm{~J} \mathrm{~mol}^{-1} \mathrm{~K}^{-1}$. Because enthalpy change $\Delta H<0$ and entropy change $\Delta S>0$, the acting force, which result in the form of the non-fluorescence complex, are mainly a hydrophobic interaction [10]. 


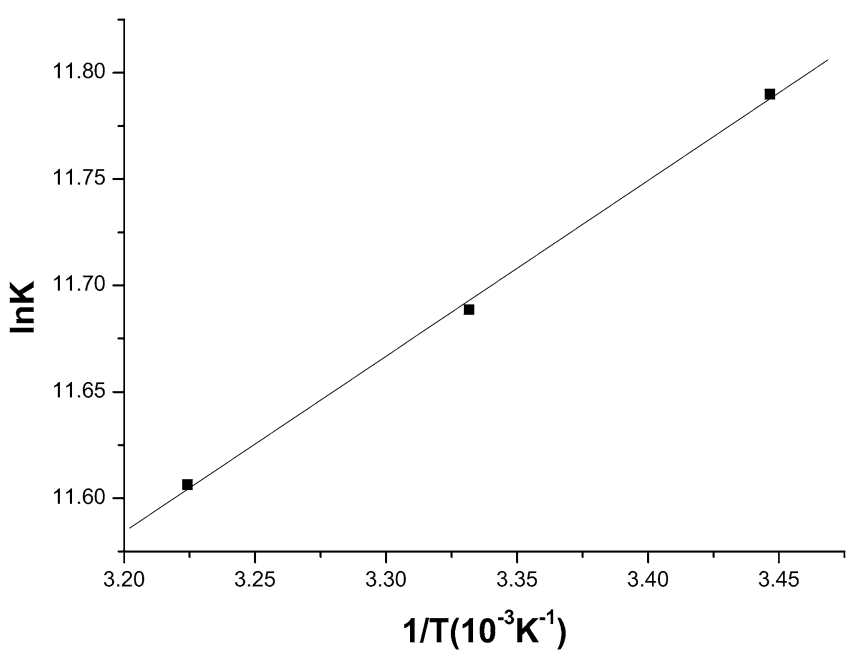

Fig. 6. Variation of $\ln K$ as a function of $1 / T$.

\subsection{The binding distance between PAL and BSA}

According to the Fơrster's theory [14], the efficiency of energy transfer, $E$, is given by:

$$
E=\frac{R_{0}^{6}}{R_{0}^{6}+r^{6}}
$$

where $r$ is the donor-acceptor distance and $R_{0}$ is the distance at $50 \%$ transfer efficiency.

$$
R_{0}^{6}=8.8 \times 10^{-25} K^{2} n^{-4} \phi J,
$$

where $K^{2}$ is the orientation factor between the emission dipole of the donor and the absorption dipole of the acceptor. The dipole orientation factor, $K^{2}$, is the least certain parameter in calculation of the critical transfer distance, $R_{0}$. Although theoretically $K^{2}$ can range from 0 to 4 , the extreme values require very rigid orientations. If both the donor and acceptor are tumbling rapidly and are free to assume any orientation, then $K^{2}$ equals $2 / 3$ [15]. If only the donor is free to rotate, then $K^{2}$ can vary from $1 / 3$ to $4 / 3$ [16]. The validity of assuming a value of $2 / 3$ has been discussed often [17-19] . Therefore, $K^{2}=2 / 3$ was used for the dynamic averaging of relative donor-acceptor positions in the PAL-BSA system; $n(=1.4)$ is the refractive index of medium [20], $\phi$ is the fluorescence quantum yield of the donor (its value was taken as 0.10 [21]) and $J$ is the overlap integral of the fluorescence emission spectra of the donor and the absorption spectra of the acceptor. $J$ is given by:

$$
J=\frac{\sum F(\lambda) \varepsilon(\lambda) \lambda^{4} \Delta \lambda}{\sum F(\lambda) \Delta \lambda},
$$

where $F(\lambda)$ is the fluorescence intensity of fluorescence reagent when the wavelength is $\lambda . \varepsilon(\lambda)$ is the molar absorbance coefficient at the wavelength of $\lambda$. From these relationships, $J, E$ and $R_{0}$ can be calculated; so the value of $r$ also can be calculated, where $J=1.2472 \times 10^{-14} \mathrm{~cm}^{3} 1 \mathrm{~mol}, R_{0}=$ $3.36 \mathrm{~nm}, r=5.01 \mathrm{~nm}$. Figure 7 is the overlap of the fluorescence spectra of BSA and the absorption 


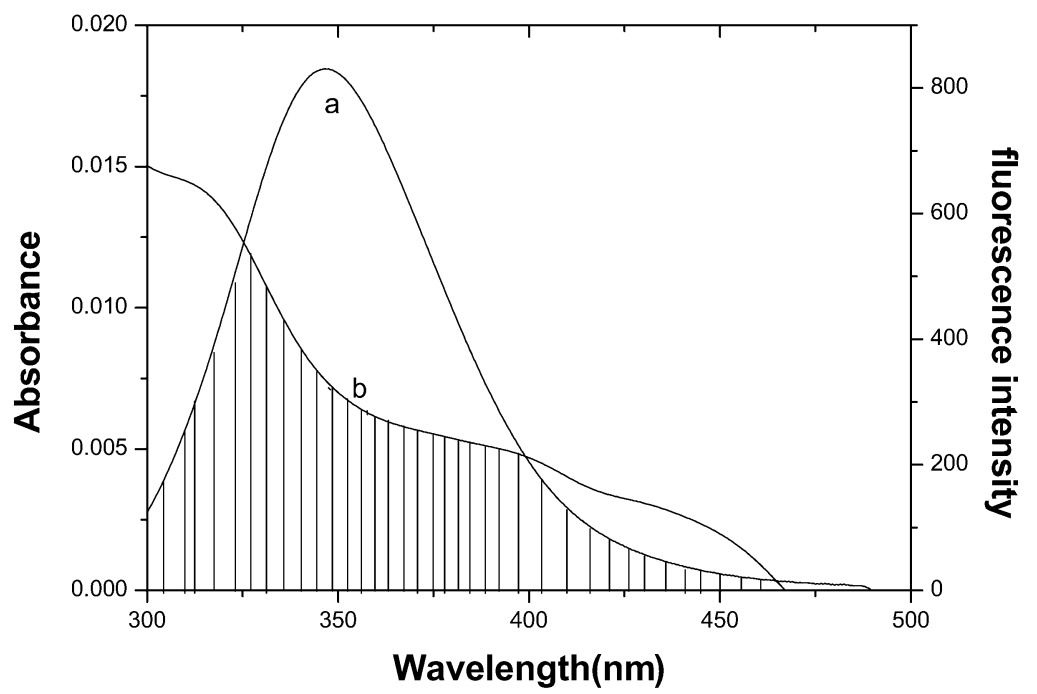

Fig. 7. The overlap of the fluorescence spectra of BSA and the absorption spectra of PAL. (a) the fluorescence spectra of BSA, (b) the absorption spectra of PAL.

spectra of PAL when the molar ratio is 1:1. For BSA, it has two tryptophan residues: Trp-214 is located in a hydrophobic fold and the additional tryptophan (Trp-135) is located on the surface of molecule. In this paper, PAL is probably bound to Trp-214 residue mainly through the hydrophobic interaction according to the thermodynamic results. However, interaction between the Trp- 135 and PAL cannot be precluded, so the distance calculated here is actually the average value between the bound PAL and the two tryptophan residues in BSA.

\section{Conclusions}

Above results indicated that paeonolum can quench the intrinsic fluorescence of BSA though binding to BSA with high affinity, and the quenching mechanisms is static quenching. In addition, the standard enthalpy change $(\Delta H)$ and the standard entropy change $(\Delta S)$ of this interaction process were calculated to be $-6.865 \mathrm{~kJ} \mathrm{~mol}^{-1}$ and $74.34 \mathrm{~J} \mathrm{~mol}^{-1} \mathrm{~K}^{-1}$, which indicated that hydrophobic forces played major role in the interaction between PA and BSA.

\section{Acknowledgements}

The authors are grateful to the National Natural Science Foundation of China (20603018) and the Natural Science Foundation of Jiangsu Education Department (04KJB150068) for their support.

\section{References}

[1] T. Peters, Biochemistry, Genetics and Medical Applications, Academic Press, San Diego, CA, 1996.

[2] D.C. Carter and J.X. Ho, in: Advances in Protein Chemistry, V.N. Schumaker, ed., vol. 45, Academic Press, San Diego, 1994. 
[3] S. Curry, H. Mandelkow, P. Brick and N. Franks, Nat. Struct. Biol. 5 (1998), 827.

[4] H.P. Rang, M.M. Dale and J. Ritter, Molecular Pharmacology, 3rd edn, Churchill Livingstone, New York, 1995.

[5] B. Nerli and G. Pico, Arch. Int. Physiol. Biochim. Biophys. 102 (1994), 5.

[6] D. Romanini, G. Avalle, B. Farruggia, B. Nerli and G. Pico, Chem. Biol. Interact. 115 (1998), 247.

[7] Z.C. Shang, P.G. Yi, Q.S. Yu and R.S. Lin, Acta Phys. Chem. Sin. 17 (2001), 48.

[8] Y.J. Jin, W.L. Li and Q.R. Wang, Biochem. Biophys. Res. Commun. 177 (1991), 474.

[9] L.J. Dong, R.P. Jia, Q.F. Li, X.G. Chen and Z.D. Hu, Analyst 126 (2001), 707.

[10] J.Q. Liu, J.N. Tian, J.Y. Zhang, Z.D. Hu and X.G. Chen, Anal. Bioanal. Chem. 376 (2003), 864.

[11] K. Zhu and S.Y. Tong, Chem. J. Chinese Universities 17 (1996), 539.

[12] W.R. Ware, J. Phys. Chem. 66 (1962), 455.

[13] C.Z. Huang, K.A. Li and S.Y. Tong, Anal.Chem. 68 (1996), 2259.

[14] T. Förster, Modern Quantum Chemistry, O. Sinanoglu, ed., vol. 3, Academic Press, New York, 1996.

[15] T. Förster, Discuss. Faraday Soc. 27 (1959), 7.

[16] C.W. Wu and L. Stryer, Proc. Natl. Acad. Sci. USA 69 (1972), 1104.

[17] J.R. Lakowica, Principles of Fluorescence Spectroscopy, 2nd edn, Kluwer/Plenum, New York, 1999.

[18] Z. Hillel and C.W. Wu, Biochemistry 15 (1976), 2105.

[19] L. Stryer, Annu. Rev. Biochem. 47 (1978), 819.

[20] S. Kasai, T. Horie and S. Awazu, J. Pharm. Sci. 76 (1987), 387.

[21] F.L. Cui, J. Fan, J.P. Li and Z.D. Hu, Bioorganic Med. Chem. 12 (2004), 151. 


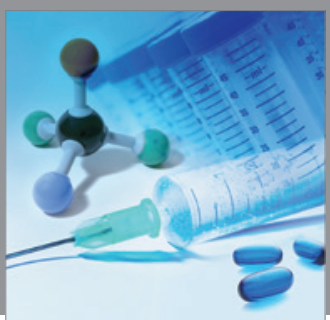

International Journal of

Medicinal Chemistry

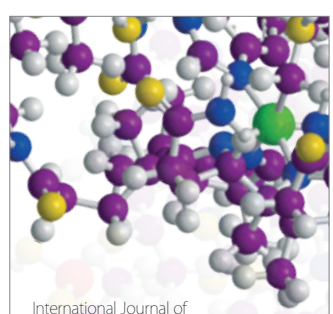

Carbohydrate Chemistry

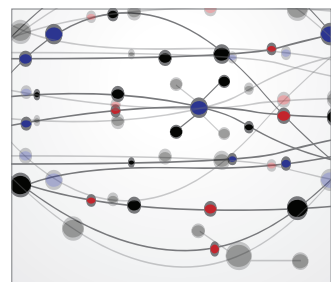

The Scientific World Journal
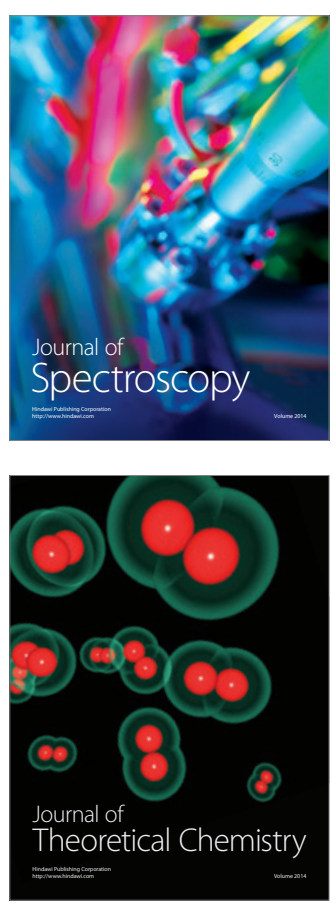
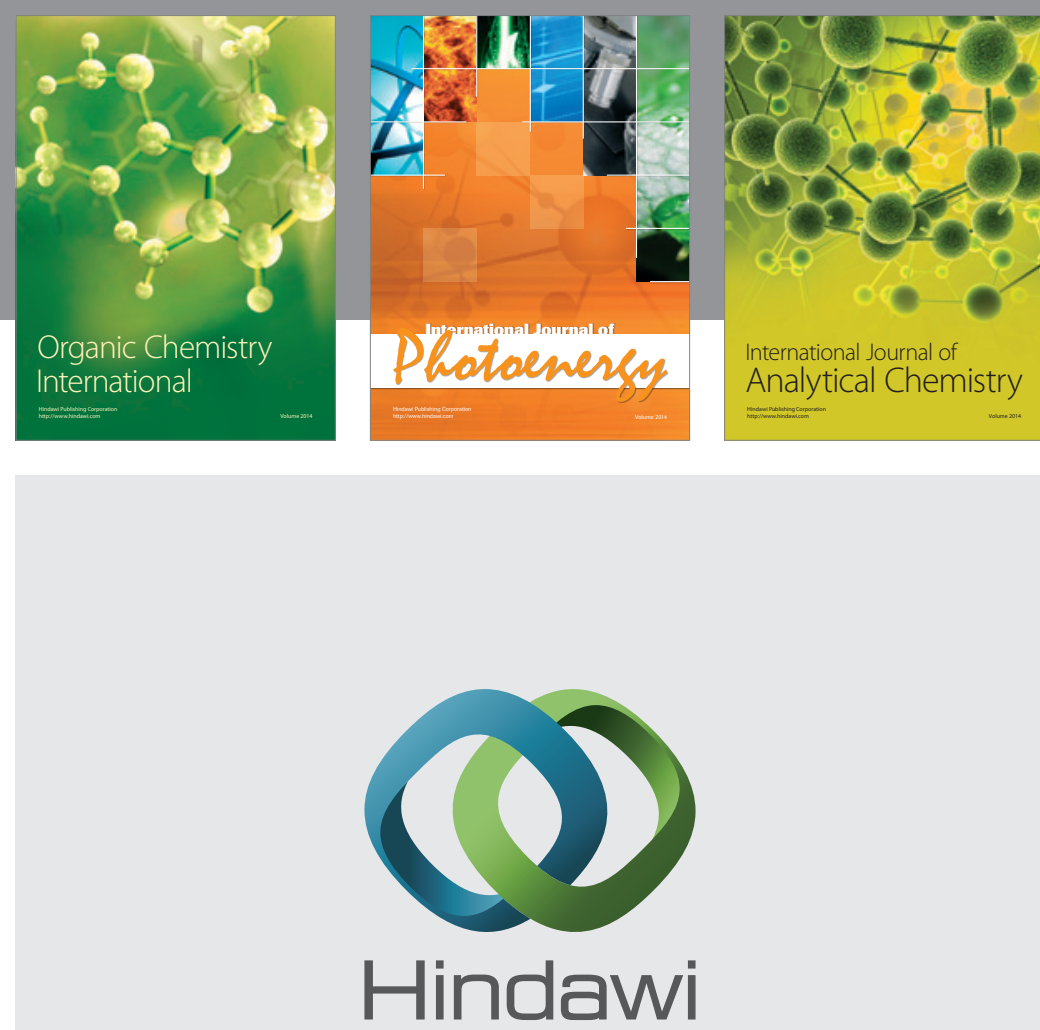

Submit your manuscripts at

http://www.hindawi.com
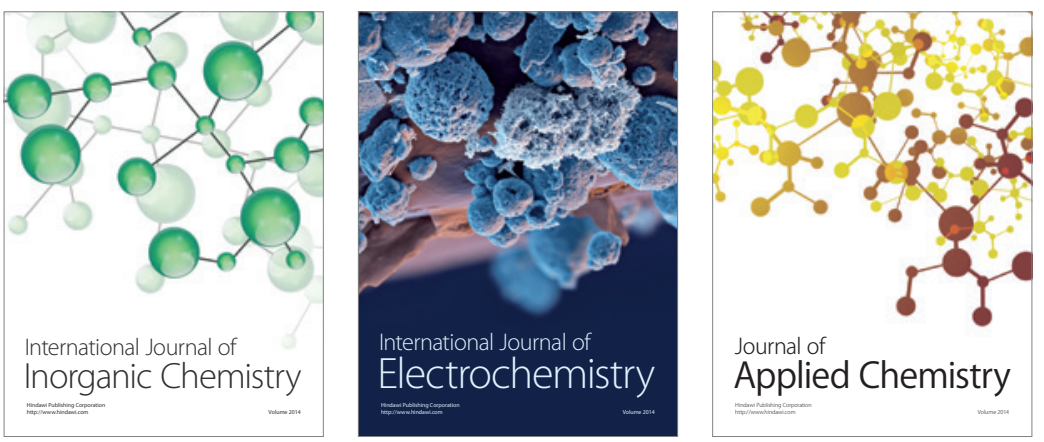

Journal of

Applied Chemistry
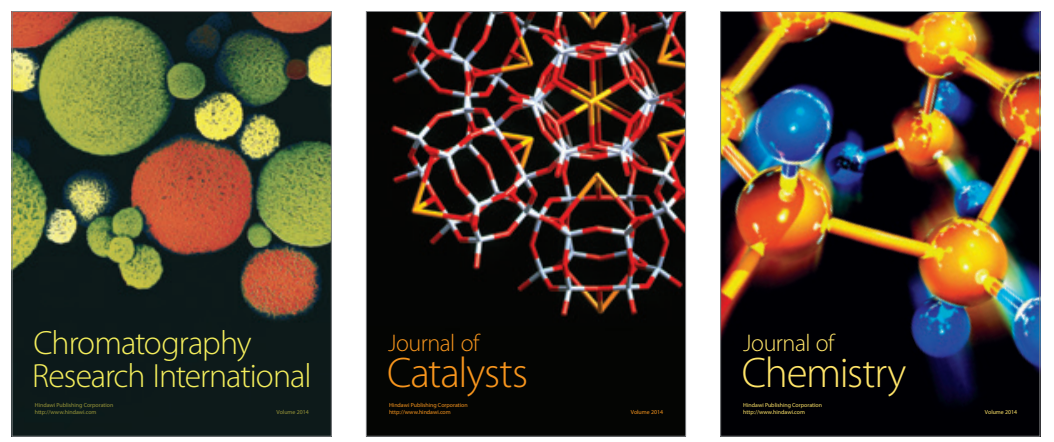
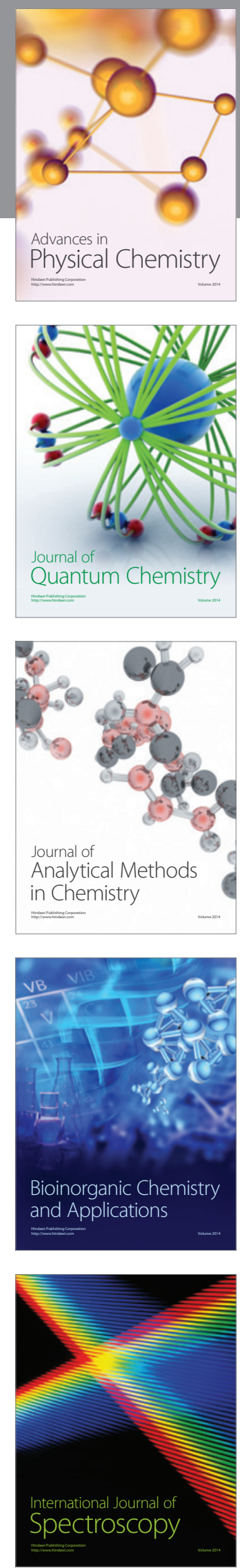